\title{
A new minimum fluorescence parameter, as generated using pulse frequency modulation, compared with pulse amplitude modulation: $\boldsymbol{F}_{\boldsymbol{\alpha}}$ versus $\boldsymbol{F}_{\mathrm{o}}$
}

\author{
A. Harrison Wright • John M. DeLong • \\ Jeffrey L. Franklin • Rajasekaran R. Lada • \\ Robert K. Prange
}

Received: 18 December 2007/Accepted: 20 May 2008/Published online: 31 July 2008

(C) The Author(s) 2008

\begin{abstract}
The minimum fluorescence parameter $\left(F_{\alpha}\right)$, generated using the new pulse frequency modulation (PFM) technology, was compared with the minimum fluorescence parameter $\left(F_{\mathrm{o}}\right)$, generated by pulse amplitude modulation (PAM), in response to a reversible low-oxygen stress in 'Honeycrisp' TM (HC) apples (Malus domestica) and an irreversible osmotic stress induced by water loss in two grape (Vitis spp.) cultivars ('L'Acadie' (LAc) and 'Thompson Seedless' (TS)). The minimum fluorescence values produced by both fluorometer types in response to a reversible low-oxygen stress in apples were indistinguishable: both $F_{\mathrm{o}}$ and $F_{\alpha}$ increased when $\mathrm{O}_{2}$ levels were lowered below the anaerobic compensation point (ACP); when gas levels returned to normoxia both parameters dipped below, then returned to, the original fluorescence baseline. The two parameters also responded similarly to the irreversible osmotic stress in grapes: in both cultivars, $F_{\alpha}$ and $F_{\mathrm{o}}$ first decreased before reaching an inflection point at approximately $20 \%$ mass loss and then increased towards a second inflection point. However, the two parameters were not analogous under the irreversible osmotic stress; most notably, the relative $F_{\alpha}$ values appeared to be lower than $F_{\mathrm{o}}$ during the later stages of dehydration. This was likely due to the influence of the $F_{\mathrm{m}}$
\end{abstract}

A. H. Wright $(\bowtie) \cdot$ J. M. DeLong · J. L. Franklin · R. K. Prange Atlantic Food and Horticulture Research Centre, Agriculture and Agri-Food Canada, Kentville, NS, Canada B4N 1J5 e-mail: wrighth@agr.gc.ca

R. R. Lada

Nova Scotia Agricultural College, Truro, NS, Canada B2N 5E3 parameter and an overestimation of $F_{\alpha}$ when measuring the fluorescence from healthy and responsive chloroplasts as found in grapes experiencing minimal water loss, but not in grapes undergoing moderate to severe dehydration. An examination of the data during a typical PFM scan reveals this fluorometer system may yield new fluorescence information with interesting biological applications.

Keywords Apple Chlorophyll $a$ fluorescence . Controlled atmosphere $\cdot$ Fluorometry $\cdot$ Grapes
Abbreviations
ACP Anaerobic compensation point
CA Controlled atmosphere
$D \quad$ LED duty cycle
$f \quad$ LED pulse frequency
$F \quad$ Relative chlorophyll fluorescence
$F_{\alpha} \quad$ PFM approximation of minimum fluorescence
$F^{\prime \prime}(f) \quad$ Second derivative of the function $F(f)$
FIRM Fluorescence interactive response monitor
$F_{\mathrm{m}} \quad$ Maximum fluorescence
$F_{\mathrm{o}} \quad$ Minimum fluorescence
$F_{\mathrm{v}} \quad$ Variable fluorescence
$F_{\mathrm{v}} / F_{\mathrm{m}} \quad$ PS II quantum efficiency
PAM Pulse amplitude modulation
PFM Pulse frequency modulation
PPFD Photosynthetic photon flux density
PSII Photosystem II
$q_{\mathrm{N}} \quad$ Non-photochemical quenching
$q_{\mathrm{P}} \quad$ Photochemical quenching
$\tau \quad$ Pulse width
$T \quad$ Pulse period
$V(f) \quad$ The location of the vertex, in terms of $f$, of the function $F(f)$ 


\section{Introduction}

A new fluorometer and software system (HarvestWatch ${ }^{\mathrm{TM}}$ 2003) developed by Satlantic Inc., Halifax, Nova Scotia, in conjunction with Agriculture and Agri-Food Canada (AAFC) uses pulse frequency modulation (PFM) to generate a novel derivation of minimum fluorescence and may be an untapped source of new fluorescence information. The PFM fluorometer was initially developed to determine the anaerobic compensation point (ACP) in fruits and vegetables destined for controlled atmosphere (CA) storage (Prange et al. 2003; DeLong et al. 2004; Prange et al. 2007; Burdon et al. 2008) and has found applications ranging from minimizing the incidence of scald in apples (Zanella et al. 2005) to maintaining kiwifruit firmness (Lallu and Burdon 2007). Many other potential stress physiology applications remain to be explored.

Lowering oxygen levels below the ACP in plants induces a stress that may be reversed with little damage, if the oxygen is increased above the ACP within a short period of time (Prange et al. 2003; DeLong et al. 2004). The physiological changes that occur in grapes (Vitis spp.) as they dehydrate over a long period of time induce osmotic stress and irreversible damage to the grape with a reduction in quantum efficiency (Wright et al. 2008).

Pulse amplitude modulation (PAM) fluorometry has produced a large number of fluorescence investigations and practical applications (Schreiber 2004). Minimum fluorescence $\left(F_{\mathrm{o}}\right)$ is measured with a low intensity far-red light $\left(\approx 6 \mathrm{nmol} \mathrm{m} \mathrm{m}^{-2} \mathrm{~s}^{-1}\right.$ at $\left.660 \mathrm{~nm}\right)$, while the maximum fluorescence $\left(F_{\mathrm{m}}\right)$ is measured using a high intensity white light saturation pulse $\left(\approx 10,000 \mu \mathrm{mol} \mathrm{m} \mathrm{m}^{-2} \mathrm{~s}^{-1}\right.$ at 400-700 nm). For plants measured under dark-adapted conditions, it is assumed that non-photochemical quenching $\left(q_{\mathrm{N}}\right)$ is zero for the measurements of both $F_{\mathrm{o}}$ and $F_{\mathrm{m}}$. The low intensity of the pulsed far-red light theoretically ensures that photosystem II (PSII) reaction centres are open (i.e. photochemical quenching $\left.\left(q_{\mathrm{P}}\right)=1\right)$ during the measurement of $F_{\mathrm{o}}$, while the highly intense saturation pulse used in generating $F_{\mathrm{m}}$ closes all PSII reaction centres $\left(q_{\mathrm{P}}=0\right)$ (van Kooten and Snel 1990). The variable fluorescence $\left(F_{\mathrm{v}}\right)$ is the difference between $F_{\mathrm{m}}$ and $F_{\mathrm{o}}$, while the quantum efficiency of PSII photon capture may be approximated by dividing $F_{\mathrm{v}}$ by $F_{\mathrm{m}}$ (DeEll et al. 1999).

In a PFM fluorometer, the amplitude of the light probe is fixed, but the frequency of the light emission is altered. In each PFM sensor, four red $635 \mathrm{~nm}$ light emitting diodes (LEDs) surround a photodiode with a cut-off filter to reject the LED excitation. This arrangement can be used to scan a relatively large surface area capable of detecting small fluorescence changes in more than one leaf, fruit or vegetable simultaneously. During each PFM scan, the duty cycle
(D) (i.e. $D=\tau T^{-1}$ where $\tau=$ pulse width and $T=$ pulse period) of the four LEDs is gradually increased over a number of increments over a period of time by decreasing $T$ while $\tau$ remains fixed. The increasingly shorter $T$ translates into a range of pulse frequencies $(f)$ over the course of a scan (Prange et al. 2007). The pulse width, duty cycle, frequency and scan time settings may be manipulated by the user (HarvestWatch $^{\mathrm{TM}}$ operator manual). The increase in $f$ results in an increase of the photosynthetic photon flux density (PPFD). Typical scans employ a 9-cm sensorsample distance and induce a range of $\approx 0.01-10 \mu \mathrm{mol}$ $\mathrm{m}^{-2} \mathrm{~s}^{-1}$ of red light at the sample surface (Prange et al. 2007); however, varying the sensor-sample distance will affect the sample surface PPFD. Contrary to the saturationpulse PAM method, which uses two distinct PPFDs to measure the $F_{\mathrm{o}}$ and $F_{\mathrm{m}}$, the PFM fluorometer measures the relative fluorescence $(F)$ over a gradient of PPFDs. The measured $F$ is a function of $f$. As the $f$ and PPFD increase, the $F$ reaches an " $F_{\mathrm{m}}$-like" parameter [it cannot be considered a true measurement of $F_{\mathrm{m}}$ since the conditions required for an $F_{\mathrm{m}}$ measurement (i.e. $q_{\mathrm{P}}=0$ and $q_{\mathrm{N}}=0$ ) are potentially violated; $q_{\mathrm{N}}$ is likely induced due to the length of the scan (58 s) and the PPFD achieved by the LEDs most probably falls short of the intensity required to close all the PSII reaction centres]. A second-order polynomial $\left(F=a \cdot f^{2}+b \cdot f+F_{\alpha}\right)$ is fitted to the data, where the extrapolated $y$-intercept $\left(F_{\alpha}\right)$ value, which has been generated using thousands of readings over the course of a scan and which has been found to be extremely sensitive to stress-induced physiological changes, represents the theoretical value of the fluorescence intensity when the PPFD equals zero (Prange et al. 2007). In other words, $F_{\alpha}$ represents the amount of fluorescence present when all the PSII reaction centres are open and photochemical quenching is at a maximum; $F_{\alpha}$ is therefore thought to approximate $F_{\mathrm{o}}$. However, the question of whether $F_{\alpha}$ is truly analogous to $F_{\mathrm{o}}$ has not been fully scrutinized and data directly comparing the two systems have never been published. For certain applications, the HarvestWatch ${ }^{\mathrm{TM}}$ PFM system has advantages over traditional PAM technology: the fluorometer units are less expensive to manufacture; the scan surface area is far greater than most available PAM fluorometers; several fluorescence sensors may be linked together; measurements may be automated and the PFM $F_{\alpha}$ parameter often demonstrates a greater sensitivity to physiological stresses than the PAM $F_{\mathrm{o}}$ parameter.

The objectives of this study were to: 1 . compare the minimum fluorescence parameter $\left(F_{\alpha}\right)$ (generated by a PFM fluorometer) with the minimum fluorescence parameter $\left(F_{\mathrm{o}}\right)$ (generated using a PAM fluorometer) in response to reversible low-oxygen stress in apples, and to irreversible damage induced by osmotic stress in grapes; and 2 . demonstrate how $F_{\alpha}$ is derived. 


\section{Materials and methods}

$F_{\alpha}$ versus $F_{\mathrm{o}}$ during low-oxygen stress in apples

Six, 41 clear plastic respiration jars were each filled with approximately $1 \mathrm{~kg}$ (i.e. 5-6 apples) of 'Honeycrisp' TM (HC) apples in a two-factor, randomized experiment. Three jars were placed inside each of three PAM fluorometer units (OS-900 prototype, Opti-Sciences Inc., Tyngsboro, MA., USA) and the fluorescence was measured hourly. The OS-900 prototype was developed specifically to measure a large surface area using PAM technology; it measures $F_{\mathrm{o}}$ and $F_{\mathrm{m}}$, but is not fitted with an actinic light needed for quenching analysis. The fluorescence in the remaining three jars was monitored hourly using three PFM sensors. Since both $F_{\mathrm{o}}$ and $F_{\alpha}$ are relative terms, the values were normalized (i.e. normalization $=($ datum point $/$ data set average $) \times 100)$. The air flow of all six jars was controlled; ambient air $\left(20.9 \% \quad \mathrm{O}_{2}\right.$ and $0.03 \% \quad \mathrm{CO}_{2}$ ) flowed through each jar at $\approx 35 \mathrm{ml} \mathrm{min}$. All work was performed in a dark room held at a constant temperature of $3.5^{\circ} \mathrm{C}$. The apples in each jar were dark-adapted for several hours before fluorescence measurements commenced. An exhaust line of equal length was run from each jar through a port leading outside the room so the flow (digital flow meter J\&W Scientific, Inc., Folsom, CA, USA) and gas levels (GCS150 Dual Analyser, Gas Control Systems Inc., Sparta, MI, USA) in each jar could be measured without influencing the fluorescence readings. The fluorescence was measured in all eight jars for $14 \mathrm{~h}$ in order to establish a fluorescence baseline, after which the flow of ambient air was turned off and a nitrogen purge $\left(\approx 100 \mathrm{ml} \mathrm{min}^{-1}\right.$ of $\left.100 \% \mathrm{~N}_{2}\right)$ was performed for $7.5 \mathrm{~h}$ (i.e. hours 14-21.5). The $\mathrm{N}_{2}$ was then turned off and the flow of ambient air through the jars was restored. Fluorescence was monitored for an additional $13.5 \mathrm{~h}$ (i.e. hours 21.5-35) after the low-oxygen stress to establish a post-stress baseline. The average minimum fluorescence for both the PAM and PFM fluorometers were compared over four, key 2-h periods: pre-low- $\mathrm{O}_{2}$ baseline (hours 4-6), low- $\mathrm{O}_{2}$ peak (hours 19-21), post-low- $\mathrm{O}_{2}$ recovery (hours 23-25) and post-low- $\mathrm{O}_{2}$ baseline (hours 32-34). A two-factor, balanced analysis of variance (ANOVA) $\left(\right.$ Minitab $^{\circledR}$ Release 15, 2007) and a least-squared means comparison (SAS Release 8.0, 1999) were used to compare normalized fluorescence averages between the fluorometers and the four key periods. Data were checked for constant variance and normality. The average standard deviation (expressed as a \%) of the fluorescence baseline prior to the $\mathrm{N}_{2}$ purge (hours 0-14) was compared between the two fluorometers using a $t$-test. Significance was defined as $P<0.05$.
$F_{\alpha}$ versus $F_{\mathrm{o}}$ during osmotic stress in grapes

Six clusters of 'Thompson Seedless' (TS) grapes (Vitis vinifera), obtained from the local supermarket and of South American origin, were divided into two treatments in a one-factor, randomized experiment. Each cluster of grapes started with a mass of approximately $240 \pm 50 \mathrm{~g}$ and were individually suspended from a wire and placed on a digital scale (PK-4, Denver Instrument Company, Arvada, CO., USA). Three cluster/scale systems were placed inside three PAM fluorometer units (Fig. 1b) (the grapes remained on the scales for the duration of the experiment); a typical point-and-click PAM unit was not used in this study since it had been determined that this design is sensitive to chlorophyll concentration in grapes (Wright et al. 2008). The remaining three clusters were monitored with PFM fluorometer sensors positioned to the left and right of each cluster for a total of six sensors (Fig. 1a). All six systems were dehydrated in a dark room for 32 days $(\approx 60 \%$ mass loss) under ambient room conditions (temperature $=$ $23 \pm 1{ }^{\circ} \mathrm{C} ; \mathrm{RH}=35 \pm 5 \%$ ). The fluorescence on each cluster of grapes was measured hourly. For the PFM system, the left and right sensor measurements were staggered (i.e. left measurement at hour 1 , right measurement at hour 2) to avoid light-induced artefacts. The PFM $F_{\alpha}$ values for each cluster were averaged to partially imitate the OS-900 PAM fluorometer system, which internally averages three sensors to generate one $F_{\mathrm{o}}$ value for each measurement. The mass of each cluster was recorded daily. Both $F_{\mathrm{o}}$ and $F_{\alpha}$ values were normalized. Individual grape cluster mass loss $(\%)$ versus minimum fluorescence plots were created. Regression analyses in conjunction with basic calculus were used to compare the average location of the inflection points (in terms of mass loss) between the two fluorometer systems. A one-factor ANOVA (Minitab ${ }^{\circledR}$ Release 15) was used to compare the average inflection point locations generated by the two different fluorometer systems. Significance was defined as $P<0.05$.

A second and third replicate of the above experiment was performed with eight clusters of 'L'Acadie' (LAc) (hybrid) grapes (product of Nova Scotia) per replicate. Each cluster of grapes had an initial mass of approximately $95 \pm 15 \mathrm{~g}$. Due to the smaller relative size of the LAc compared with the TS grapes (individual berries weighed approximately $1.2 \mathrm{~g}$ and $5.8 \mathrm{~g}$, respectively), the LAc fruit dehydrated more quickly (Dreier et al. 2000) and were held in a dark room under ambient conditions (see above) for 18 days ( $\approx 70 \%$ mass loss).

Dissection of the PFM $F_{\alpha}$ derivation

To better understand how the $F_{\alpha}$ parameter is derived and influenced, the raw data generated during a PFM fluorometer 
Fig. 1 (a) Dehydrating grapes: PFM side-scan (sensors were placed $10 \mathrm{~cm}$ from sample), (b) dehydrating grapes: PAM side-scan (each unit was an equilateral triangle:

length $=44 \mathrm{~cm}$, height $=20 \mathrm{~cm}$; sensor-sample distance was $10 \mathrm{~cm}$ ), (c) low-oxygen apples: PFM side-scan and (d) dehydrating grapes: PFM sensor 'float' ('float' base $=160 \mathrm{~cm}^{2}$ )
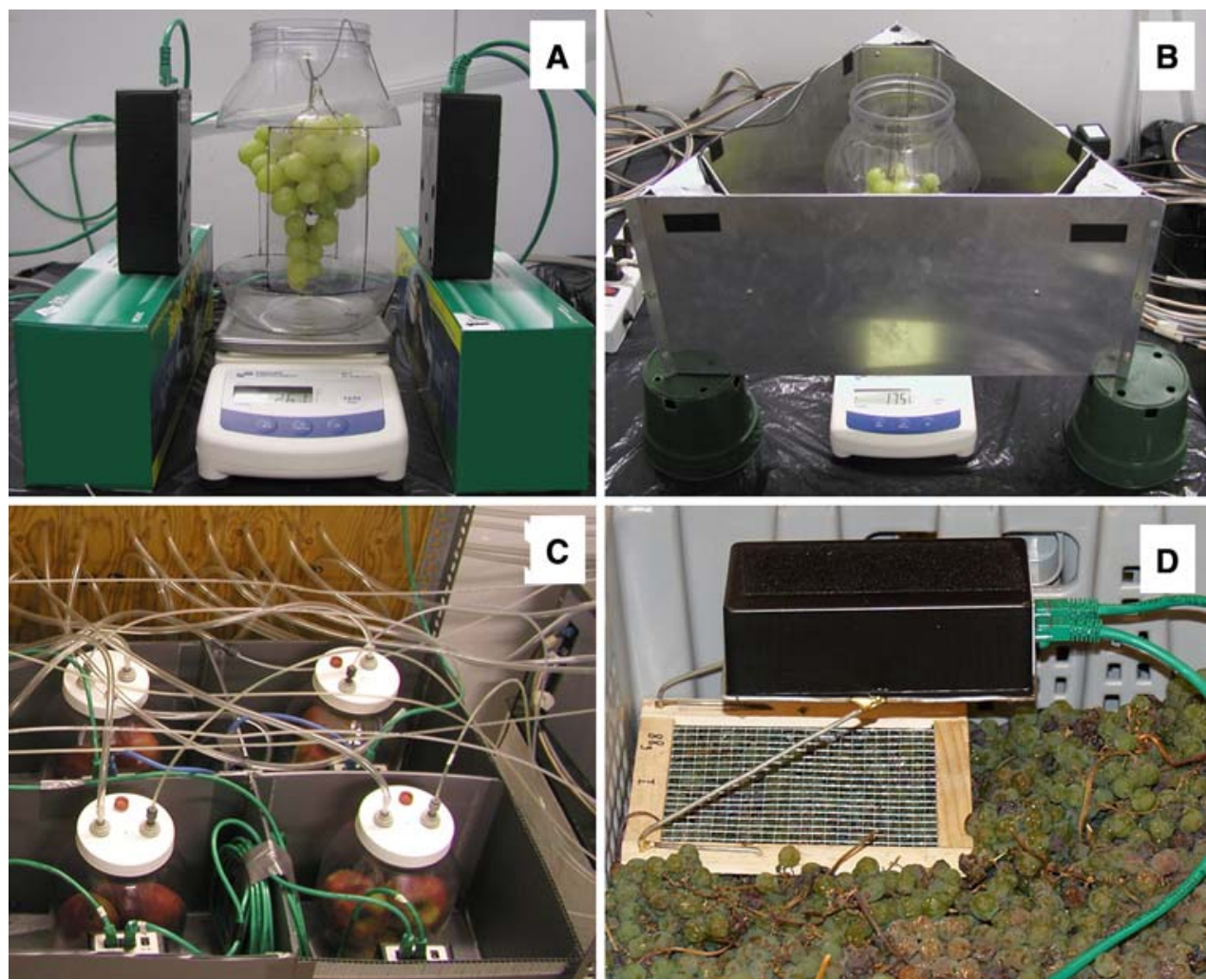

scan were decoded in order to generate $f$ versus $F$ plots for key points of interest for the apples exposed to low-oxygen stress and the grapes subjected to osmotic stress. The PFM sensor settings were the same for all measurements and used a $D$ range of $0.000366-0.05868$ and a $f$ range of $\approx 0.10$ $15 \mathrm{kHz}$ with a $58 \mathrm{~s}$ scan time.

For the low-oxygen apple trials, three consecutive scans at each of the four points of interest (i.e. 1. pre-low- $\mathrm{O}_{2}$ baseline, 2. low- $\mathrm{O}_{2}$ peak, 3. post-low- $\mathrm{O}_{2}$ recovery and 4 . post-low- $\mathrm{O}_{2}$ baseline) were generated. Regression analysis was performed on these plots (Minitab ${ }^{\circledR}$ Release 15) so that the nature of the second order polynomial response relative to the data, the goodness-of-fit, and the $y$-intercept (i.e. $F_{\alpha}$ ), for each plot could be analysed.

For the grape dehydration trials, a PFM sensor was placed in a carrier 'float' that rested on top of a hamper filled with TS grapes as they dehydrated (temperature $=$ $23 \pm 1{ }^{\circ} \mathrm{C} ; \mathrm{RH}=35 \pm 5 \%$ ) (Fig. 1d) in order to generate a stronger, more coherent fluorescence signal; four experimental replications were used. This apparatus was deemed more applicable to a commercial setting; it also ensured that the sensor interacted with a solid surface of drying grapes and the distance between the grapes and the sensor remained constant. Scans were performed every $2 \mathrm{~h}$ until the grapes achieved a weight loss of $80 \%$. Over the course of dehydration, five key points of interest were identified on the resulting mass loss versus $F_{\alpha}$ plots [i.e. 1. the initial $F_{\alpha}$ value, measured under inadequate dark-adaptation (approximately $10 \mathrm{~min}$ of dark-adaptation was allowed prior to the first scan); 2. first measurement following adequate dark-adaptation time; 3 . first primary inflection point; 4. second primary inflection point and 5 . a point after the second primary inflection point]. Three consecutive $f$ versus $F$ plots for each of the five points of interest were analysed as described above for the apple trial.

The $f$ versus $F$ plots for the low-oxygen apple work and the grapes undergoing osmotic stress were used to demonstrate the potential of deriving other new PFMbased fluorescence parameters besides $F_{\alpha}$. The second derivative $\left(F^{\prime \prime}(f)\right)$ (analogous to line curvilinearity), the vertex location $(V[f])$ and the height of the fitted quadratic in the $f$ versus $F$ plots, as well as an " $F_{\mathrm{v}}$-like" parameter (i.e. vertex height subtract $F_{\alpha}$ ) were discussed as examples of potential physiologically rich PFM-based parameters that may aid in quantifying changing $f$ versus $F$ curve dynamics.

\section{Results}

A side-scan approach was employed by both the PAM and PFM fluorometers when measuring low-oxygen stress in apples (PAM apple setup not shown) and osmotic stress in grapes (Fig. 1a-c). A fluorometer 'float' (Fig. 1d) was employed to collect data used to examine the grape dehydration PFM $f$ versus $F$ plots. 
$F_{\alpha}$ versus $F_{\mathrm{o}}$ during low-oxygen stress in apples

There did not appear to be any difference between $F_{\alpha}$ and $F_{\mathrm{o}}$ in response to low-oxygen stress in apples (Table 1): each fluorescence parameter sustained a steady baseline during initial normoxia, showed a slight increase immediately after the $\mathrm{N}_{2}$ purge began, rose sharply after $\mathrm{O}_{2}$ levels

Table 1 The average normalized minimum fluorescence values over the four stages of the low-oxygen spike using both PAM and PFM fluorometers

\begin{tabular}{|c|c|c|}
\hline Stage & $\operatorname{PAM}\left(F_{\mathrm{o}}\right)$ & $\operatorname{PFM}\left(F_{\alpha}\right)$ \\
\hline 1. Pre-low- $\mathrm{O}_{2}$ baseline & $98.9^{\mathrm{bA}}$ & $99.1^{\mathrm{bA}}$ \\
\hline 2. Low- $\mathrm{O}_{2}$ peak & $111.2^{\mathrm{aA}}$ & $111.3^{\mathrm{aA}}$ \\
\hline 3. Post-low- $\mathrm{O}_{2}$ recovery & $96.5^{\mathrm{cA}}$ & $96.9^{\mathrm{cA}}$ \\
\hline 4. Post-low- $\mathrm{O}_{2}$ baseline & $98.8^{\mathrm{bA}}$ & $99.2^{\mathrm{bA}}$ \\
\hline
\end{tabular}

Averages with similar lowercase letter groupings within a column are not significantly different $(P<0.05)$. Averages with similar uppercase letter groupings within a row are not significantly different $(P<0.05)$ were reduced below the $\mathrm{ACP}$ and then fell below the original fluorescence baseline once normoxia was re-established before returning to pre-stress levels (Fig. 2).

The standard deviation of the fluorescence baseline was significantly higher when using the PAM $( \pm 2.20 \%)$ compared with the PFM system $( \pm 0.19 \%)(P<0.001)$. The PAM $F_{\mathrm{m}}$ parameter was little-affected by sub-ACP oxygen, but declined slightly before quickly recovering when conditions were returned to normoxia (data not shown).

$F_{\alpha}$ versus $F_{\mathrm{o}}$ during osmotic stress in grapes

The minimum fluorescence parameters of both the PAM (Fig. 3a) and PFM (Fig. 3b) fluorometers generated similar sigmoidal curves in response to water loss in the TS grapes; however, the PFM fluorescence signal appeared to decrease more sharply after the second inflection point compared with the PAM signal. The initial increase at the onset of the $F_{\alpha}$ data (Fig. 3b) was not included in the regression
Fig. 2 The fluorescence spike induced by low-oxygen as measured by a (a) PAM and (b) PFM fluorometer. Raw data are shown in grey; black lines indicate averaged values. The symbol $(\boldsymbol{\Delta})$ indicates when the atmospheric air was turned off and the $\mathrm{N}_{2}$ purge began and the symbol $(\boldsymbol{\nabla})$ indicates when the $\mathrm{N}_{2}$ flow was turned off and the flow of atmospheric air was returned

Fig. 3 The averaged relationship between mass loss (TS) and the normalized minimum fluorescence values (a) $F_{\mathrm{o}}^{1}$ and (b) $F^{2}$. Raw data are shown in grey; fitted response curves for averaged data appears as solid line; fitted response curves for individual clusters appear as dashed lines. ${ }^{1} \mathrm{y}=-6.8 \times 10^{-4} \mathrm{x}^{3}+$ $0.070 \mathrm{x}^{2}-1.86 \mathrm{x}+107$, $\mathrm{R}^{2}=0.83 ;{ }^{2} \mathrm{y}=-1.0 \times 10^{-3} \mathrm{x}^{3}$ $+0.093 x^{2}-2.36 x+115$, $\mathrm{R}^{2}=0.89$
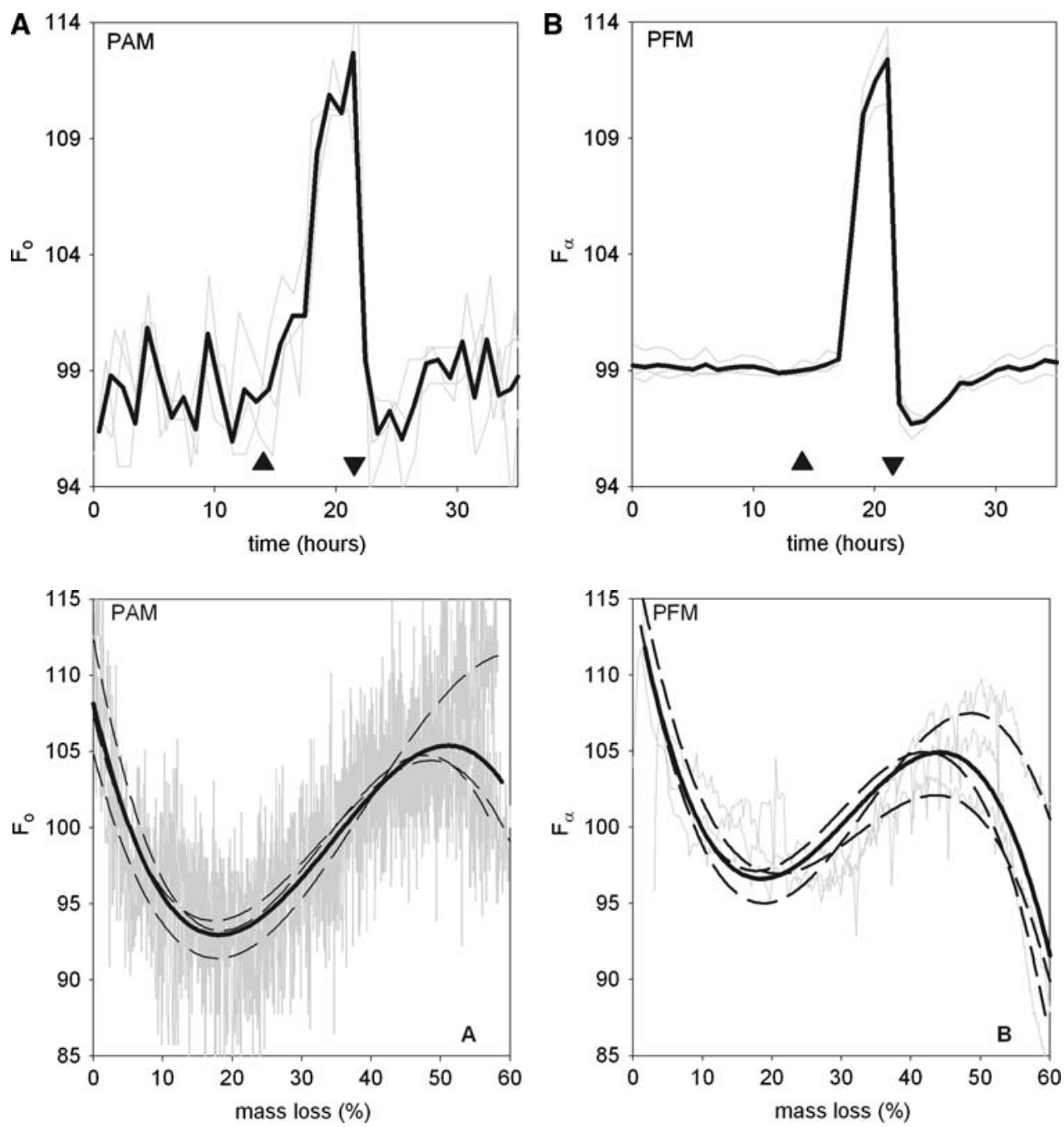
Table 2 The average location of the first and second major inflection point in the mass loss versus fluorescence for both PAM and PFM fluorometer systems using TS grapes

\begin{tabular}{lllcl}
\hline & $n$ & & $\begin{array}{l}\text { First inflection } \\
(\text { mass loss }(\%))\end{array}$ & $\begin{array}{l}\text { Second inflection } \\
(\text { mass loss }(\%))\end{array}$ \\
\hline $\operatorname{PAM}\left(F_{\mathrm{o}}\right)$ & 3 & Avg. & $17.6^{\mathrm{a}}$ & $54.4^{\mathrm{a}}$ \\
& & SD & 0.5 & 10.8 \\
$\operatorname{PFM~}\left(F_{\alpha}\right)$ & 3 & Avg. & $16.8^{\mathrm{a}}$ & $44.4^{\mathrm{a}}$ \\
& & SD & 3.0 & 2.5 \\
\hline
\end{tabular}

Averages with similar letter groupings within a column are not significantly different $(P<0.05)$

analysis, as it was believed to be an artefact of inadequate dark-adaptation.

There was no difference between the two fluorometers in the average location (\% mass loss) of both the first and second major inflection points found on the individual cluster response curves $(P=0.651$ and $P=0.193$, respectively) (Table 2). However, in both cases the statistical assumption of constant variance (general guideline: $0.5<\mathrm{SD}<2$ ) was compromised and the small sample size meant conversion factors would be ineffective.

The minimum fluorescence parameters of the two different fluorometers generated dissimilar curvilinear fitted responses to water loss in LAc berries (Fig. 4). The PAM fluorometer generated a quadratic response curve that appeared to approach, but did not reach, a second major inflection point, within the dehydration range measured (Fig. 4a). The PFM signal was sigmoidal and reached a well-defined second inflection point at approximately $65 \%$ mass loss (Fig. 4b). There was a greater propensity for the

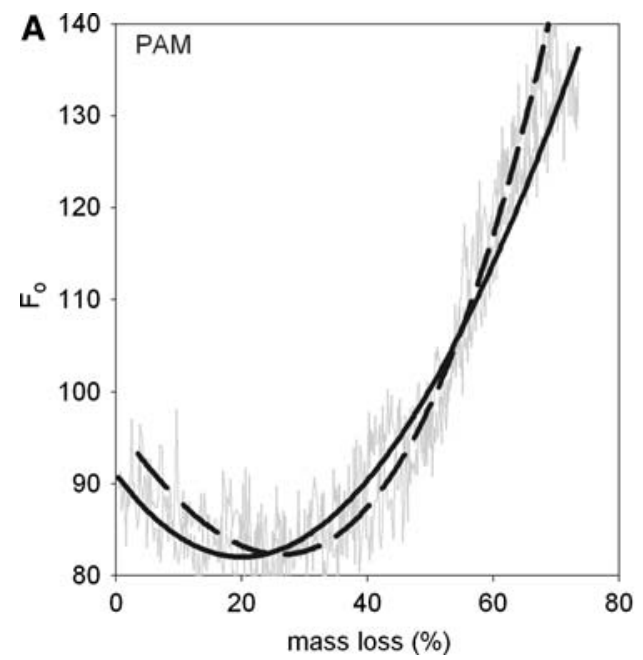

Fig. 4 The averaged relationship between mass loss (LAc) and the normalized minimum fluorescence values (a) $F_{\mathrm{o}}$ : rep. 1 (solid) ${ }^{1}$, rep. 2 (dashed) $)^{2}$ and (b) $F$ : rep. 1 (solid) ${ }^{3}$, rep. 2 (dashed) ${ }^{4}$. Raw averaged data are shown in grey; each represents averaged data from four
PFM minimum fluorescence signal to decrease compared with the PAM, exhibited by the steeper decline towards the initial inflection point and the occurrence of a second inflection point in the PFM data (Fig. 4). The two data sets generated using the LAc grapes were not combined so the repeatability of the generated relationships could be observed (Fig. 4; Table 3).

Although the coefficients of determination for the fitted response curves were relatively high for both fluorometer types and cultivars, the minimum fluorescence signal appeared to be more erratic when using the PAM compared with the PFM fluorometer (Figs. 2 and 3). In the PFM data, the few deviations of the $F_{\alpha}$ signal from the fitted response curve can largely be attributed to low-intensity light exposure when the door to the dark-room was slightly opened momentarily (Figs. $3 b$ and $4 b$ ). This occurred even though door-openings were consistently timed to occur

Table 3 The average location of the first major inflection point in the mass loss versus fluorescence for both PAM and PFM fluorometer systems using LAc grapes

\begin{tabular}{|c|c|c|c|c|}
\hline & $n$ & & $\begin{array}{l}\text { First inflection } \\
\text { (mass loss }(\%) \\
\text { rep. 1) }\end{array}$ & $\begin{array}{l}\text { First inflection } \\
\text { (mass loss }(\%) \\
\text { rep. 2) }\end{array}$ \\
\hline \multirow[t]{2}{*}{$\operatorname{PAM}\left(F_{\mathrm{o}}\right)$} & 4 & Avg. & $22.4^{\mathrm{a}}$ & $26.5^{\mathrm{a}}$ \\
\hline & & SD & 6.0 & 5.1 \\
\hline \multirow[t]{2}{*}{$\operatorname{PFM}\left(F_{\alpha}\right)$} & 4 & Avg. & $24.7^{\mathrm{a}}$ & $26.5^{\mathrm{a}}$ \\
\hline & & SD & 7.7 & 5.1 \\
\hline
\end{tabular}

Averages with similar letter groupings are not significantly different $(P<0.05)$

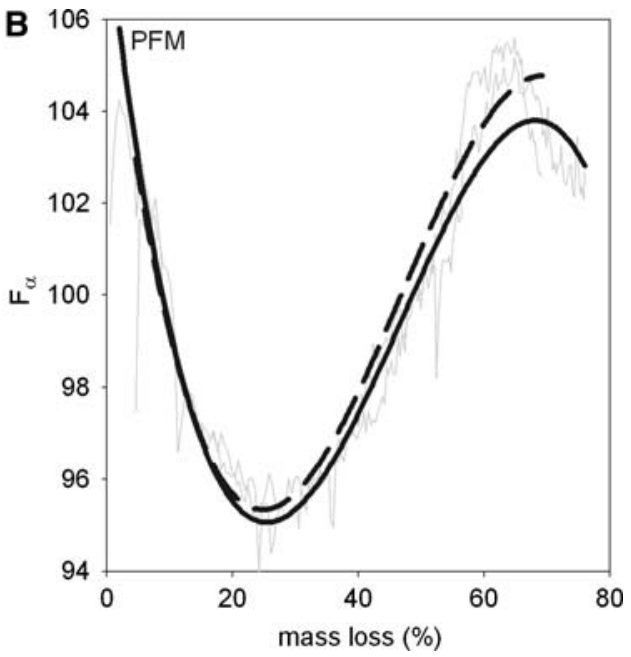

clusters. ${ }^{1} y=4.8 \times 10^{-4} x^{3}+0.025 x^{2}-0.931 x+91.2, \mathrm{R}^{2}=0.97$; ${ }^{2} y=1.6 \times 10^{-4} x^{3}+0.013 x^{2}-1.00 x+96.9, \mathrm{R}^{2}=0.96 ;{ }^{3} y=2.2 \times$ $10^{-4} x^{3}+0.031 x^{2}-1.16 x+108, \mathrm{R}^{2}=0.96 ;{ }^{4} y=2.6 \times$ $10^{-4} x^{3}+0.036 x^{2}-1.33 x+110, \mathrm{R}^{2}=0.95$ 
directly after a scan. Opening the door did not appear to similarly affect the PAM $F_{\mathrm{o}}$ signal.

The average location of the first primary inflection point in the mass loss versus minimum fluorescence relationship was similar between PAM and PFM systems and between replications when using the LAc grapes (Table 3). Although there was no occurrence of a second major inflection point in the PAM data, the location of this point was comparable $(P=0.701)$ between replications for the PFM data: rep. $1=67.9 \%$; rep. $2=71.5 \%$.

Dissection of the PFM $F_{\alpha}$ derivation

For both the low-oxygen apple and grape dehydration trials, $F_{\alpha}$ values generated using the raw data and regression analysis matched those generated by the HarvestWatch ${ }^{\mathrm{TM}}$ software (data not shown). In both the apple and grape work (with the exception of point 1 for the latter), the three fitted response curves at each point of interest were highly similar (Figs. 5 and 6). The mass loss versus $F_{\alpha}$ plot generated with the sensor 'float' was similar to that of the sidescan approach; the dynamics of each possessed the same 5 key points of interest (Figs. 3b and 6a).

For the low-oxygen-stressed apples (Fig. 5a), the $f$ versus $F$ plots from the pre- and post- low- $\mathrm{O}_{2}$ baselines (points 1 and 4) showed similar dynamics (Fig. 5b). $F$ first increased dramatically and then continued to increase at a more moderate rate relative to increased $f$ (where $f$ is proportional to PPFD) to form a concave curve (i.e. a negative $F^{\prime \prime}(f)$ ). Interestingly, under low- $\mathrm{O}_{2}$ conditions,
$F$ was relatively high under low PPFD and then was severely quenched before increasing moderately under increasing PPFD (Fig. 5c) to form a convex curve (i.e. a positive $F^{\prime \prime}(f)$ value). During the post-low- $\mathrm{O}_{2}$ recovery period (point 3) (Fig. 5d), the $f$ versus $F$ plots appeared similar to the pre- and post-low- $\mathrm{O}_{2}$ baseline scans, but $F$ was more highly quenched at the higher PPFD values (i.e. a decreased " $F_{\mathrm{v}}$-like" parameter).

For the grape dehydration work, the $f$ versus $F$ relationships, especially those occurring at points 2 and 3 , displayed the dynamics of a typical Kautsky curve (Fig. 6) (where $f$ is proportional to time). The grape scans revealed increased quenching at the higher $f$ (i.e. increased PPFD) levels, most notable in scans performed under non-stressed conditions, compared with the apple scans (Figs. 5b and $6 \mathrm{c}$ ). At point 1 (Fig. $6 \mathrm{~b}$ ), the magnitude of $F$ was much lower in the first grape PFM measurement (time $=0 \mathrm{~h}$ ) compared with the subsequent two at 2 and $4 \mathrm{~h}$, respectively, and appeared to be less curvilinear. After reaching a maximum at point 2 (based on $F^{\prime \prime}(f)$ values), the data became less curvilinear over the course of dehydration, with the three replications at point 5 showing the lowest degree of curvilinearity (Fig. 6b-f).

The $y$-intercept (i.e. $F_{\alpha}$ ) of the fitted response curve appeared to overestimate the true minimum fluorescence values as observed in data sets exhibiting a high degree of curvilinearity (i.e. grapes measured under minimal water loss) (e.g. Fig. 6c, d).

The $f$ versus $F$ plots created for the side-scan grapes gave very similar results to those generated using the
Fig. 5 (a) A representative $\mathrm{HC}$ PFM time (hours) versus $F$ plot showing four points of interest, (b-d) corresponding $f$ versus $F$ plots for points $1-4$. The individual data points, response curve and equation, $F^{\prime \prime}(f)$ and $V$ $[f]$ of the first scan $\left(\mathrm{O}_{-}\right)$at each point of interest is shown, while only the response curve $(--)$ is shown for the second and third scans at each point of interest
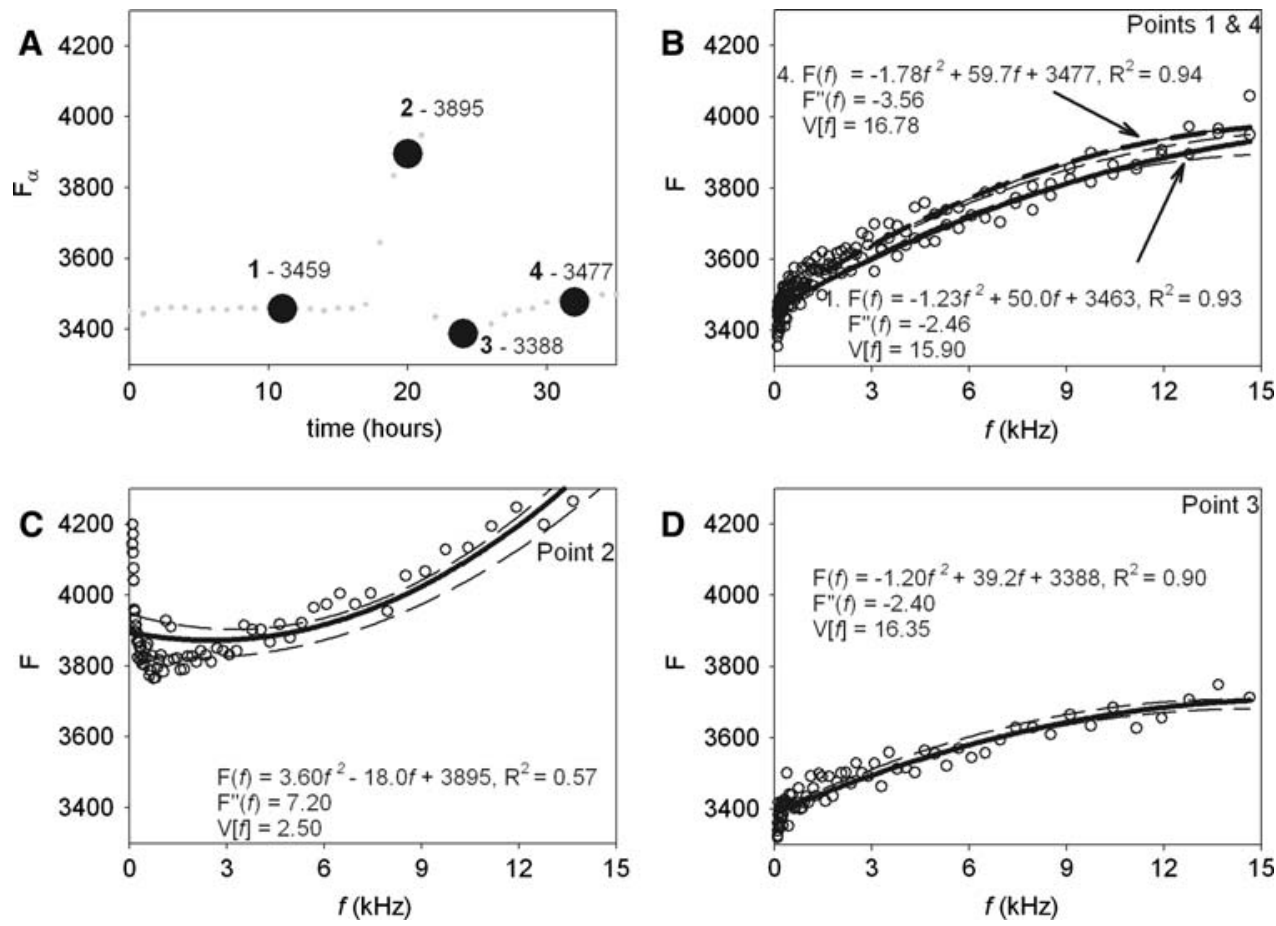
Fig. 6 (a) a representative TS PFM mass loss versus $F$ plot showing five points of interest, (b-f) corresponding five $f$ versus $F$ plots. Each plot shows the data and response curve for three consecutive scans. The individual data points, response curve and equation, $F^{\prime \prime}(f)$ and $V$ $[f]$ of the first scan $\left(\mathrm{O}^{-}\right)$at each point of interest are shown, while only the response curve (- - ) is shown for the second and third scans at each point of interest
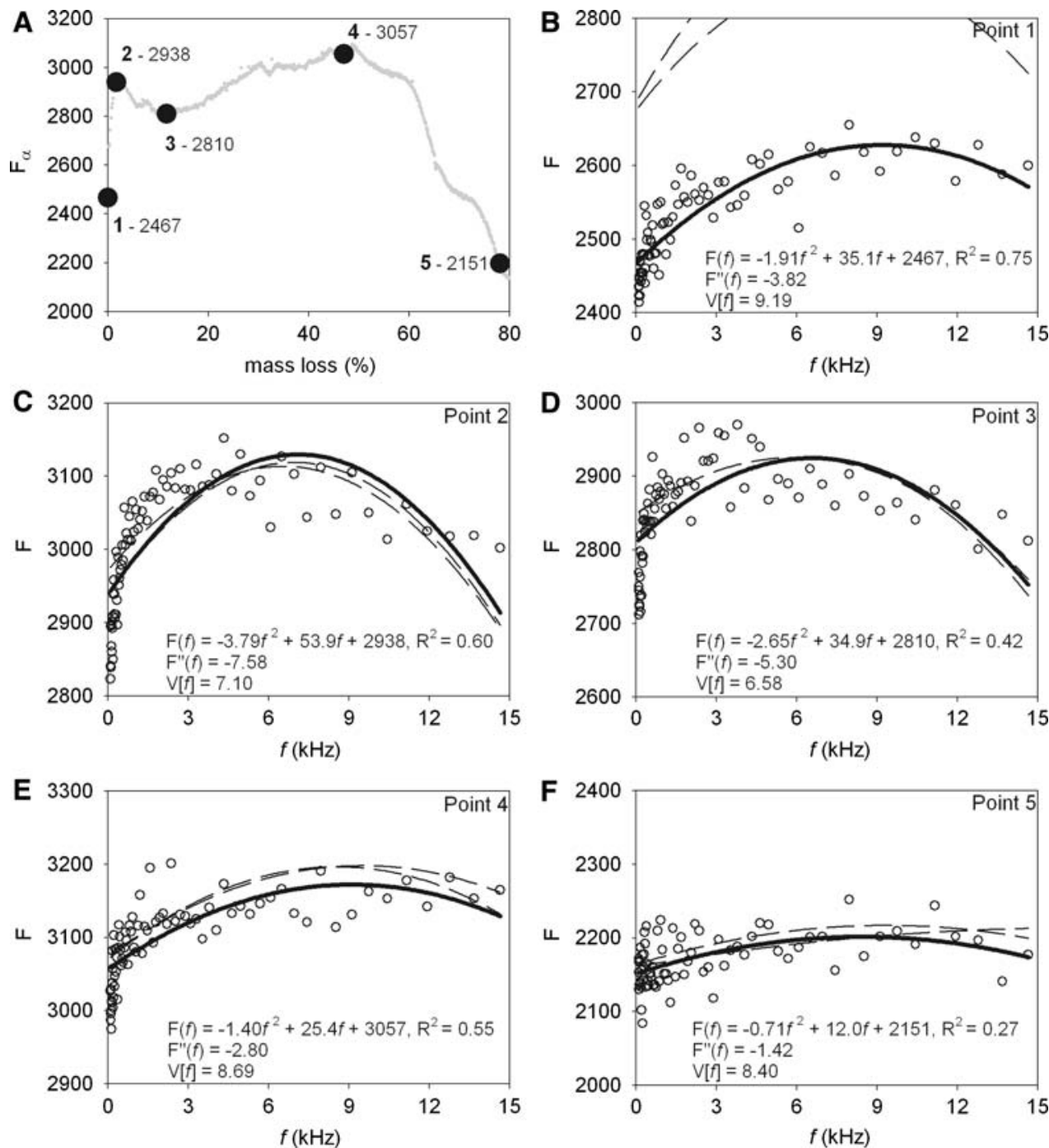

sensor 'float', but showed weaker $F$ values (data not shown).

\section{Discussion}

Although sub-ACP oxygen levels have been shown to increase both $F_{\mathrm{o}}$ (Prange et al. 2002) and $F_{\alpha}$ (Prange et al. 2003; DeLong et al. 2004) in fruits and vegetables, these are the first data showing a direct comparison. For the reversible low-oxygen stress on apples, the PAM and PFM minimum fluorescence parameters were indistinguishable (Table 1). In the grape dehydration trials, the differences between the two fluorometers did not affect the location of the first inflection point in the mass loss versus minimum fluorescence relationship for either cultivar (Tables 2 and 3), but did affect the occurrence of a second inflection point when using LAc (Fig. 4). The greater propensity for the PFM minimum fluorescence value to decline relative to grape mass loss compared with the PAM value likely occurred due to: 1) sensitivity to a decreasing $F_{\mathrm{m}}$ factor, and 2 ) an overestimation of $F_{\alpha}$ during periods of high curvilinearity in the $f$ versus $F$ plots.

Both $F_{\mathrm{m}}$ and $F_{\mathrm{v}}$ have been shown to significantly decrease in both grape cultivars over the course of dehydration (Wright et al. 2008). If the $F_{\alpha}$ values were influenced by this decreasing $F_{\mathrm{m}}$ parameter [there is evidence for this based on the similarity of the mass loss versus $F_{\alpha}$ and mass loss versus vertex height (i.e. " $F_{\mathrm{m}}$-like") plots (data not shown)], it could be responsible for lower relative $F_{\alpha}$ compared with $F_{\mathrm{o}}$ values with respect to mass loss. An ancillary experiment with the PAM fluorometer showed that the $F_{\mathrm{m}}$ parameter decreased sharply under inadequate dark-adaptation conditions, while $F_{\mathrm{o}}$ remained relatively unchanged (data not shown). The $F_{\mathrm{m}}$ value is lower in the presence of light than in a dark-adapted sample as a result of non-photochemical quenching $\left(q_{\mathrm{N}}\right)$ (van Kooten and Snel 1990). Under conditions of inadequate dark-adaptation, the grape 
PFM $F_{\alpha}$ value was relatively low, resulting in a spike in fluorescence as the grapes became dark-adapted (Figs. 3b, $4 \mathrm{~b}$ and $6 \mathrm{~b}$ ). This sudden increase at the onset of each trial was not apparent when using the PAM fluorometer (Figs. 3a and $4 \mathrm{a}$ ). The apparent increased sensitivity of the PFM $F_{\alpha}$ value to inadequate dark-adaptation, compared with the PAM $F_{\mathrm{o}}$ value, may be due, at least in part, to the influence of a decreasing $F_{\mathrm{m}}$ parameter.

In the grape dehydration trials, an overestimation of $F_{\alpha}$ during periods of high curvilinearity (in the $f$ versus $F$ plots) may also influence $F_{\alpha}$ downwards over the course of dehydration. When grapes were turgid, healthy and measured under adequate dark-adaptation (as shown in Fig. 6c), the fluorescence rose steeply to a maximum and then quenched slowly, dynamics typical of a classic Kautsky curve. When applying a second-order polynomial to these data, the sharp incline in fluorescence observed at the lower $f$ values causes the $y$-intercept to be overestimated (Fig. 6c). However, as the grapes dehydrate, the efficiency of their photosystems declines (Wright et al. 2008), the sharpness of the incline in fluorescence at the low $f$ values is reduced, and the overestimation of the $y$-intercept also decreases, which may be interpreted (in terms as $F_{\alpha}$ values) as a factor influencing the minimum fluorescence parameter downwards relative to mass loss (Fig. 6c-f). For example, at point 5 (Fig. 6f) the $y$-intercept for each of the three respective replications shown is in the middle of the data point clusters observed around the low $f$ values.

The difference in the minimum fluorescence parameter generated by the two different types of fluorometers was more apparent in the LAc grapes compared with the TS. A third factor, chlorophyll degradation, will also affect fluorescence levels. TS grapes undergo significant chlorophyll degradation during dehydration, while LAc grapes do not (Wright et al. 2008). Chlorophyll content is positively correlated with minimum fluorescence (Smillie et al. 1987; Toivonen and DeEll 1998). Therefore, when using the TS grapes, the relationship between grape mass loss and the PAM and PFM minimum fluorescence parameters appeared more similar because of the commonality of chlorophyll degradation, a factor that likely influenced both $F_{\mathrm{o}}$ and $F_{\alpha}$ downwards. The difference between the two fluorometer types was more apparent when the LAc cultivar, which was less affected by chlorophyll degradation, was tested. However, chlorophyll loss would have been a relative non-factor in the low-oxygen apple experiments in which the two relative minimum fluorescence parameters were indistinguishable (Fig. 2; Table 1).

The $f$ versus $F$ plots (Figs. 5 and 6 ) indicate the potential for the derivation of new PFM-based fluorescence parameters. The difference in these plots for apples and grapes under relatively non-stressed conditions (Figs. 5b and 6c) showed that $F$ was quenched at the higher frequencies in grapes to a greater degree than it was in apples. The location of the curve vertex $(V[f])$ could be used to quantify this difference [i.e. $V[f]$ occurs at a significantly lower $f$ value in the grapes $(\approx 7 \mathrm{kHz})$ compared with the apples under nonstressed conditions $(\approx 16 \mathrm{kHz})]$. The curvilinearity of the response (approximated by $F^{\prime \prime}(f)$ ) may also hold potential as a PFM-based parameter that may show a high correlation with quenching levels or photosystem integrity. A relatively large, negative $F^{\prime \prime}(f)$ value (as found in both non-stressed grapes and apples (Figs. 5b and 6c)) indicates a strong, concave, relative fluorescence response to increased $f$ (and PPFD). A relatively small, negative $F^{\prime \prime}(f)$ value may indicate a photosystem with compromised integrity (as observed in dehydrated grapes (Fig. 6f)). A positive $F^{\prime \prime}(f)$ value (as found in apple under low-oxygen conditions (Fig. 5c)) indicates a convex response to increased $f$ and may indicate there is a change in quenching. If a third-order polynomial was used on the $f$ versus $F$ plots, the goodness of fit of the fitted curve may be improved and it could increase the potential of developing new PFM-based parameters. Manipulating the PPFD, via the $f$ range and pulse width, and the scan time could be used for finding specific parameters or to calibrate the fluorometer for specific plant species. Future studies should investigate correlations between PAM $q_{\mathrm{P}}$ and $q_{\mathrm{N}}$ analysis (as well as other established PAM-based information) and new PFM-based parameters. The search for new PFM-based parameters may identify fluorescence characteristics analogous to those found using PAM technology or it may yield completely new physiologically rich information.

Although PFM $F_{\alpha}$ does not appear to be completely analogous to the PAM $F_{\mathrm{o}}$, this study demonstrated that the two parameters shared common characteristics, such as a tight correlation with grape water loss with similar inflection points around the $20 \%$ mass loss region and an indistinguishable response to short term, low-oxygen stress in apples. Already used for determining ACP in fruits and vegetables within $\mathrm{CA}$ storage, the PFM sensor may also find a commercial application in the appassimento-style dessert wine industry. The relative low-cost, multiple sensor, large scan surface area approach of the HarvestWatch $^{\text {TM }}$ PFM system makes it an attractive tool for many commercial applications. The data produced by the unique HarvestWatch $^{\text {TM }}$ PFM fluorometer probe also hold the potential for the development of new PFM-derived fluorescence parameters capable of monitoring photochemical changes in the photosystem.

Acknowledgements We would like to thank Peter Harrison and Conny Bishop for their technical assistance, Dr. Michael Hardman for mathematical resources and Dr. John Cullen, Dr. Douglas Campbell and Stephanie Bishop for reviewing this article. This is contribution no. 2354 of the Atlantic Food and Horticulture Research Centre, Agriculture and Agri-Food Canada. We gratefully acknowledge the 
Natural Sciences and Engineering Research Council of Canada for Canadian Graduate Studies doctoral funding for $\mathrm{H}$. Wright.

Open Access This article is distributed under the terms of the Creative Commons Attribution Noncommercial License which permits any noncommercial use, distribution, and reproduction in any medium, provided the original author(s) and source are credited.

\section{References}

Burdon J, Lallu N, Haynes G, McDermott K, Billing D (2008) The effect of delays in establishment of a static or dynamic controlled atmosphere on the quality of 'Hass' avocado fruit. Postharvest Biol Technol 49:61-68

DeEll JR, van Kooten O, Prange RK, Murr DP (1999) Applications of chlorophyll fluorescence techniques in postharvest physiology. Hortic Rev 23:69-107

DeLong JM, Prange RK, Leyte JC, Harrison PA (2004) A new technology that determines low-oxygen thresholds in controlledatmosphere-stored apples. HortTechnology 14:262-266

Dreier LP, Stoll GS, Ruffner HP (2000) Berry ripening and evapotranspiration in Vitis vinifera L. Am J Enol Vitic 51:340-346

Lallu N, Burdon J (2007) Experiences with recent postharvest technologies in kiwifruit. Acta Hort 753:733-740

HarvestWatch ${ }^{\mathrm{TM}}$ Operator's Manual 1.0. (2003) Satlantic Incorporated, Halifax, NS

Minitab Inc. ${ }^{\circledR}$ Release 15.1.1.0 (2007) State College, Pennsylvania, USA

Prange RK, DeLong JM, Leyte JC, Harrison PA (2002) Oxygen concentration affects chlorophyll fluorescence in chlorophyllcontaining fruit. Postharvest Biol Technol 24:201-205
Prange RK, DeLong JM, Harrison PA (2003) Oxygen concentration affects chlorophyll fluorescence in chlorophyll-containing fruit and vegetables. J Am Soc Hortic Sci 128:603-607

Prange R, DeLong J, Harrison P, Leyte J, Mclean SD, Scrutton JGE, Cullen JJ (2007) Method and apparatus for monitoring a condition in chlorophyll containing matter. US Patent 7,199, 376, 3 Apr 2007

SAS Release 8.0. (1999) Statistical analysis system. SAS Institute, Cary, NC

Schreiber U (2004) Pulse-Amplitude-Modulation (PAM) fluorometry and saturation pulse method: an overview. In:Papageorgiou GC, Govindjee (eds) Chlorophyll $a$ fluorescence, vol 19. Springer, Dordrecht, pp 279-319

Smillie RM, Hetherington SI, Nott R, Chaplin GR, Wade NL (1987) Applications of chlorophyll fluorescence to the postharvest physiology and storage of mango and banana fruit and the chilling tolerance of mango cultivars. ASEAN Food J 3:55-59

Toivonen PMA, DeEll J (1998) Differences in chlorophyll fluorescence and chlorophyll content of brocolli associated with maturity and sampling section. Postharvest Biol Technol 14: $61-64$

van Kooten O, Snel JFH (1990) The use of chlorophyll fluorescence nomenclature in plant stress physiology. Photosynth Res 25: $147-150$

Wright H, DeLong J, Lada R, Prange R (2008) The relationship between water status and chlorophyll $a$ fluorescence in grapes (Vitis spp.) Postharvest Biol Technol (in press)

Zanella A, Cazzanelli P, Panarese A, Coser M, Cecchinel M, Rossi O (2005) Fruit fluorescence response to low-oxygen stress: modern storage technologies compared to 1-MCP treatment of apple. Acta Hort 682:1535-1542 